Original research article

\title{
Rosmarinic acid suppresses inflammation, angiogenesis, and improves paclitaxel induced apoptosis in a breast cancer model via NF3 kB-p53-caspase-3 pathways modulation
}

\author{
Marwa A. Mahmoud ${ }^{1}$, Tark M. Okda ${ }^{1}$, Gamal A. Omran ${ }^{1}$, Mohammad M. Abd-Alhaseeb 2 * \\ ${ }^{1}$ Damanhour University, Faculty of Pharmacy, Department of Biochemistry, Behira, Egypt \\ 2 Damanhour University, Faculty of Pharmacy, Department of Pharmacology and Toxicology, Behira, Egypt
}

\begin{abstract}
Rosmarinic acid is a natural polyphenolic compound that is found in different plant species and used for different medicinal purposes. This study aimed to investigate the chemo-preventive effect of rosmarinic acid and evaluate its antitumor efficacy alone or in combination with Paclitaxel in breast cancer mice model. Ehrlich induced mice mammary solid tumor model was used in the study. Mice were treated with oral rosmarinic acid and intraperitoneal Paclitaxel. Inflammation, angiogenesis, and apoptosis were checked. Enzyme linked immunosorbent assay (ELISA), quantitative real time PCR, and immunohistochemical methods were performed. Rosmarinic acid used prior to tumor induction suppressed NF- $\mathrm{kB}, \mathrm{TNF}-\alpha$, vascular endothelial growth factor (VEGF) serum levels, and VEGF receptors. It also triggered apoptosis by restoring the levels of P53, Bcl-2, Bax, and caspase-3. Furthermore, in Ehrlich solid tumor mice, rosmarinic acid, and/or Paclitaxel significantly suppressed tumor growth with an increase in apoptotic markers P53 and Caspase-3 levels, and suppressed the $\mathrm{Bcl} 2 / \mathrm{Bax}$ ratio.

Rosmarinic acid exerted chemo-preventive and therapeutic potential alone or in combination with Paclitaxel. Moreover, rosmarinic acid targets numerous signaling pathways associated with breast cancer.

Keywords: Angiogenesis; Apoptosis; Breast cancer; Inflammation; Paclitaxel; Rosmarinic acid

Highlights:

- Rosmarinic acid is natural polyphenol, it has many medicinal activities.

- Rosmarinic acid has a prophylactic effect against cancer.

- Rosmarinic acid potentiates Paclitaxel therapeutic effect.

- Rosmarinic acid activity takes place through induction of apoptosis, suppression of inflammation and angiogenesis.
\end{abstract}

\section{Abbreviations:}

ELISA, Enzyme Linked Immunosorbent Assay; NF-кB, Nuclear Factor Kappa B; PTX, Paclitaxel; qRT-PCR, Quantitative Real Time Polymerase Chain Reaction; RA, Rosmarinic Acid; SEC, Solid Ehrlich Carcinoma; TNF-a, Tumor Necrosis Factor Alpha; VEGF, Vascular Endothelial Growth Factor

\section{Introduction}

Breast cancer is a global disease, and its treatment depends mainly on surgery, radiotherapy, chemotherapy, and hormonal therapy used separately or in combination (Al-Harras et al., 2016). Unfortunately, breast cancer showed significant molecular heterogeneity, which limited the traditional treatment of the disease, such as the development of resistance in cancer cells, low specificity, and negative side effects (Day et al., 2020).

The solid Ehrlich carcinoma (SEC) is used to model breast cancer. It is a mice model and has rapid proliferation, high ma- lignancy, and similar features to inflammatory breast cancer in humans (Feitosa et al., 2020).

Paclitaxel (PTX) is a natural alkaloid Food and Drug Administration (FDA) approved chemotherapeutic drug for the treatment of solid tumors like breast cancer. It is mainly extracted from the bark of the Taxus brevifolia tree. It exerts its effect by binding to tubulin, stabilizing tubulin dimmers, preventing microtubule dissociation, and inhibiting mitosis in the M-phase causing cell death (Xu et al., 2020). Although its efficacy has been approved, it has many reported limitations, including myelotoxicity, neurotoxicity, and development of drug resistance (Kang et al., 2009).

\footnotetext{
* Corresponding author: Mohammad M. Abd-Alhaseeb, Damanhour University, Faculty of Pharmacy, Department of Pharmacology and Toxicology,1 El-gomhoria Street, Behira, Egypt; e-mail: m.abdelhasseb@pharm.dmu.edu.eg http://doi.org/10.32725/jab.2021.024 
Natural herbal products are naturally occurring bioactive compounds that have different pharmacological activities and are used in the treatment of many diseases including cancer treatment. These products are promising cancer therapies, and have many advantages (Parveen et al., 2019).

Rosmarinic acid (RA) is a polyphenol extracted from many herbs. It belongs to the Lamiaceae, Poraginaceae, and Blechnaceae families (Trócsányi et al., 2020). It was widely used for medicinal purposes including antiviral, bactericidal, antioxidant, anti-inflammatory, and anticancer. It exerts its action by different mechanisms (e.g. it prevents tumor formation development, reduces lipid peroxidation by products and pro apoptotic proteins expression, reduces tumor necrosis factor- $\alpha$ (TNF- $\alpha$ ), cyclooxygenase-2 (COX-2) and IL-6 levels, modulates p65 expression and stimulates apoptosis by modifying multiple genes expression, and is involved in apoptosis regulation) making it effective in cancer treatment (Elufioye and Habtemariam, 2019; Nadeem et al., 2019).

Consequently, the current study aimed to evaluate the chemo-preventive and anticancer effect of RA alone and/or in combination with PTX in Ehrlich solid carcinoma. In addition, we aimed to investigate the underlying mechanisms and pathways of RA in breast cancer.

\section{Materials and methods}

\section{Reagents and chemicals}

RA $\geq 96 \%$ (HPLC) (RA) (Sigma-Aldrich, catalog No: 5369545G) and PTX (Sigma-Aldrich, catalog No: T7402) were used. The Ehrlich's ascites carcinoma-infected mice were purchased from the Medical Research Institute, Alexandria University (Alexandria, Egypt). All other chemicals used in the study were of analytical grades from commercial sources.

\section{Animals}

Female Swiss albino mice weighing 25-30 g were purchased from the Animal Care Unit of Alexandria University Medical Research Institute (Alexandria, Egypt).

The mice were adapted in polyethylene cages, maintained under particular laboratory conditions $\left(25^{\circ} \mathrm{C} \pm 1{ }^{\circ} \mathrm{C}\right.$ with constant relative humidity and normal dark/light cycle) with ad libitum access to food and water. The study was performed following the internationally accepted recommendations for laboratory animal use and care in accordance with the European Community guidelines (EEC Directive of 1986; 86/609/EEC), and the protocols were approved by The Animal Care and Ethical Committee at the Faculty of Pharmacy, Damanhour University (1018PB7).

\section{Tumor induction}

Ascitic fluid of Ehrlich ascites carcinoma-infected mice was withdrawn then mixed with normal saline to form a suspension of $2 \times 10^{6}$ cells and used as a source of Ehrlich carcinoma cells (Katary et al., 2019). The viability of cells were checked by a Trypan blue dye exclusion assay (Abd-Alhaseeb et al., 2013).

At the start of the experiment (day 1), $0.1 \mathrm{ml}$ of Ehrlich's ascites carcinoma suspension was injected intra-dermally on the upper left ventral side of the mice inside the mammary fat pad of mice. After one week (day 8) of inoculation with the tumor cells, tumor growth was validated using a caliper. The volume of solid tumors $\left(\mathrm{mm}^{3}\right)$ was calculated by the following equation: Tumor Volume $=$ length $\times$ width $^{2} \times 0.52$. The solid tumors were visually apparent $\left(50-100 \mathrm{~mm}^{3}\right) 7$ days after in- oculation and treatment protocols were started (Abd-Alhaseeb et al., 2014).

\section{Experimental design}

We randomized 60 mice into 6 groups: 10 mice in each group. Group 1 (normal mice): healthy mice were intradermally injected with normal saline $(0.1 \mathrm{ml} /$ mouse, i.d.) on the upper left ventral side on day 1 . They were then given oral saline ( $1 \mathrm{ml} / \mathrm{kg} /$ day, p.o.) from day 8 to the end of the experiment.

Group $2(\mathrm{RA}+\mathrm{SEC})$ : mice were given RA orally $(50 \mathrm{mg} / \mathrm{kg} /$ day, p.o.) daily for 2 weeks prior to tumor induction and this continued to the end of the experiment ( 6 weeks therapeutic period) (Rocha et al., 2015). The dose selected for RA was in a safe range as it has been reported to have very low toxicity in mice with an LD50 of $561 \mathrm{mg} / \mathrm{kg}$ (Kirchler et al., 2017).

Treatment for groups 3 to 6 started when tumor growth was confirmed on day 8 after inoculation of the tumor, and was continued for 21 days (3 weeks therapeutic period). Group 3 (SEC): mice were given oral saline ( $1 \mathrm{ml} /$ day, p.o.), and served as the positive control group. Group 4 (SEC + RA): mice were treated with oral RA (100 mg/kg/day, p.o.) (Han et al., 2018). Group 5 (SEC + PTX): mice were treated with intraperitoneal PTX injection (10 mg/kg/three times weekly, i.p.) (Ghoneum et al., 2019). Group 6 (SEC + RA + PTX): mice were treated with a combination of RA (100 mg/kg/day, p.o.) daily and PTX (10 mg/kg/three times weekly, i.p.).

At the end of the experiment, the animals were euthanized by ether inhalation for approximately $2 \mathrm{~min}$ in a transparent acrylic jar (Anjum et al., 2018) and blood samples were collected from the orbital sinus. The mice were then sacrificed by cervical dislocation and solid tumors were dissected and weighed.

\section{Tumor assessment}

Tumor growth was determined using a digital caliper after the first week to confirm growth. Tumor weight was determined at the end of the experiment using a digital balance scale.

\section{Enzyme-linked immunosorbent assay (ELISA)}

Whole-blood samples were collected, and the samples were centrifuged at $3000 \mathrm{rpm}$ for $10 \mathrm{~min}$ at $4{ }^{\circ} \mathrm{C}$ to collect serum. The serum protein levels of vascular endothelial growth factor (VEGF) and tumor necrosis factor-alpha (TNF- $\alpha$ ) were measured with the corresponding ELISA kits from MyBioSource, Inc., CA, USA (Catalog No.: MBS703609, MBS355371 respectively) according to the manufacturer's protocols.

\section{Quantitative real-time PCR (qRT-PCR)}

Tumor tissues were used for total RNA extraction using TRIzol $^{\circledR}$ (Invitrogen; Thermo Fisher Scientific, Inc., Waltham, MA, USA), according to the manufacturer's protocol.

cDNA was synthesized from extracted RNA in the presence of SYBR Green and Master mix (Thermo Fisher Scientific, Inc., Waltham, MA, USA) using the manufacturer's protocol.

P53, Bax, and Bcl-2 gene expressions were analyzed by quantitative PCR with primers present (Table 1). qRT-PCR amplification was performed under the following thermal cycle conditions: initially at $95{ }^{\circ} \mathrm{C}$ for $10 \mathrm{~min}$, then $95{ }^{\circ} \mathrm{C}$ for $15 \mathrm{~s}$ repeated for 40 cycles for denaturation, then $56{ }^{\circ} \mathrm{C}$ for $15 \mathrm{~s}$ for annealing with primer, then $72{ }^{\circ} \mathrm{C}$ for $30 \mathrm{~s}$ for extension; final extension step at $72{ }^{\circ} \mathrm{C}$ for $10 \mathrm{~min}$. The experiments were performed three times. The relative expression levels of P53, Bax, and Bcl-2 compared to GAPDH in each sample were calculated according to the $2^{-\triangle \Delta C q}$ method (Livak and Schmittgen, 2001). 
Table 1. Primer sequence used for $q R T-P C R$

\begin{tabular}{lc}
\hline Primer sequence (5'-3') & Gene (Amplicon size) \\
\hline F: 5'GTATCGGACGCCTGGTTAC3' & GAPDH (128 bp) \\
R: 5'CTTGCCGTGGGTAGAGTCAT3' & P53 (137 bp) \\
\hline F: 5'CCCCTGTCATCTTTTGTCCCT3' & Bax (100 bp) \\
R: 5'AGCTGGCAGAATAGCTTATTGAG3' & Bcl-2 (95 bp) \\
\hline F: 5'GTCTCCGGCGAATTGGAGAT3' & \\
R: 5'ACCCGGAAGAAGACCTCTCG3' & \\
\hline F: 5'CATCGCCCTGTGGATGACTG3' & \\
R: 5'GGCCATATAGTTCCACAAAGGC3' & \\
\hline NCBI reference sequence was used for the design of the primers of \\
GAPDH, P53, Bax, and Bcl-2.
\end{tabular}

\section{Histopathological investigation and immunohistochemical analysis}

After scarification of the mice, tumor was collected, washed, and fixed in $10 \%$ neutral buffered formalin for $24 \mathrm{~h}$ at $4{ }^{\circ} \mathrm{C}$. It was then processed for paraffin sectioning used for Hematoxylin and Eosin (H \& E) staining for histopathological examination and immunostaining.

The immunostaining was performed with polyclonal antibody against Nuclear Factor kappa B/p65 (NFk B/P65) (BiossWoburn, Massachusetts, U.S.A. catalog No: bs-0465R), VEGF polyclonal antibody (BioGenex, USA, catalog No: AR483-5R), P53 polyclonal antibody (Bioss Woburn, Massachusetts, U.S.A. catalog No: bs-0033R), Bcl-2 polyclonal antibody (GeneTex, USA catalog No: GTX100064), caspase3 (cleaved) polyclonal antibody (biocare medical, CA, USA catalog No: PP 229 AA), and using dye 3, 3'-diaminobenzidine (DAB) [Sigma-Aldrich ${ }^{\circledR}$ (MO, USA)].

The tumor sections were microscopically investigated with a digital image capture system (Olympus BX41, camera model no Olympus DP20 at 40X, USA), examined for specific staining, and images were acquired. Quantitative evaluation of the tumor was conducted using ImageJ analysis (ImageJ/Fiji 1.46) downloaded from the NIH website (https://imagej.nih.gov/ij/ download.html).

\section{Statistical analysis}

Data were analyzed using GraphPad Prism 3.0 software (GraphPad Software, USA) using the one-way ANOVA analysis of variance test followed by the Tukey post-hoc test. Results are presented as the mean \pm standard deviation (SD), and a $P$-value $<0.05$ was considered significant. All experiments were performed at least three times.

\section{Results}

\section{Tumor weight}

There was suppression in tumor weight in both chemo-preventive and treated groups. Mice that received RA prior to tumor induction showed significant $(p<0.001)$ reduction in the tumor weight compared to SEC mice. Mice treated with RA + PTX showed the most significant $(p<0.001)$ decrease in tumor weight compared to SEC and their monotherapies (Fig. 1).

\section{Serum levels of TNF- $\alpha$ and VEGF}

SEC-bearing mice showed the highest levels of TNF- $\alpha$ and VEGF in serum. The prophylactic group showed a more significant reduction in TNF- $\alpha$ and VEGF serum levels than SEC mice

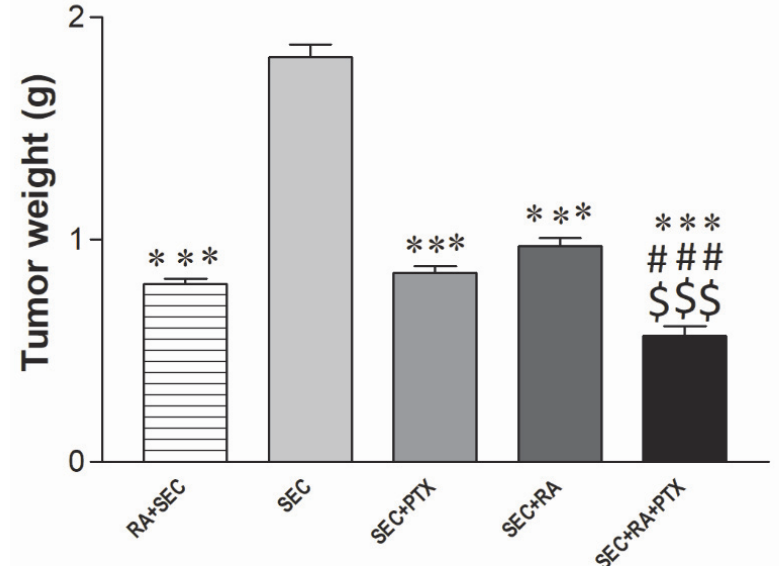

Fig. 1. Tumor weight in Ehrlich solid tumor bearing mice. Data are expressed as the mean $\pm \operatorname{SD}(n=6)$. Data were analyzed using one-way ANOVA followed by Tukey post-hoc test. RA: Rosmarinic acid; SEC: Solid Ehrlich Carcinoma; PTX: Paclitaxel. * Significant difference from SEC. \$ Significant difference from SEC + PTX group. \# Significant difference from SEC + RA group (*** or \#\#\# or $\$ \$ \$$ represent $p<0.001$ ).

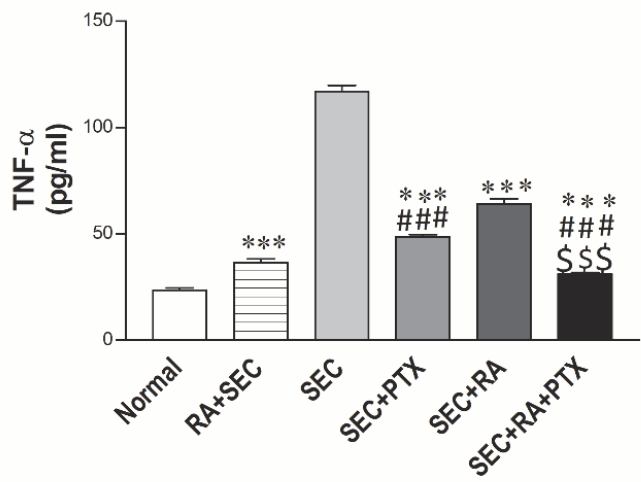

(a)

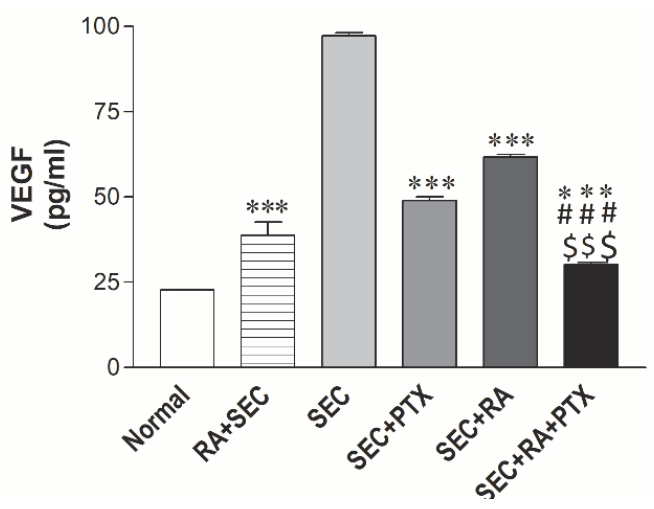

(b)

Fig. 2. The effect of treatment on serum levels of TNF- $\alpha$ and VEGF. Effect of RA, PTX and their combination on (a) TNF- $\alpha$ level, (b) VEGF level. Data are expressed as the mean $\pm \operatorname{SD}(n=6)$. Data were analyzed using one-way ANOVA followed by Tukey post-hoc test. RA: Rosmarinic acid; SEC: Solid Ehrlich Carcinoma; PTX: Paclitaxel. * Significant difference from SEC. \$ Significant difference from SEC + PTX group. \# Significant difference from SEC + RA group (*** or \#\#\# or $\$ \$ \$$ represent $p<0.001)$.

(Fig. 2). Moreover, PTX and RA significantly decreased serum levels of TNF- $\alpha$ and VEGF. SEC-bearing mice treated with a combination of PTX and RA showed the most significant decrease in TNF- $\alpha$ and VEGF levels in the serum in comparison to that of SEC and their monotherapies. 


\section{P53, Bax, and Bcl-2mRNA expressions}

RA chemoprevention, treatment alone and with PTX significantly increased the gene expression of P53 and Bax (Figs 3a and $\mathrm{Bb}$ ). The P53 gene expression of RA + SEC, SEC + PTX, SEC + RA, SEC + RA + PTX were 1.35, 3.49, 2.16, and 4.09, respectively compared to that of SEC bearing mice and the Bax fold gene expressions were 2.36, 1.92, 1.82, and 2.58, respectively compared to that of SEC bearing mice.

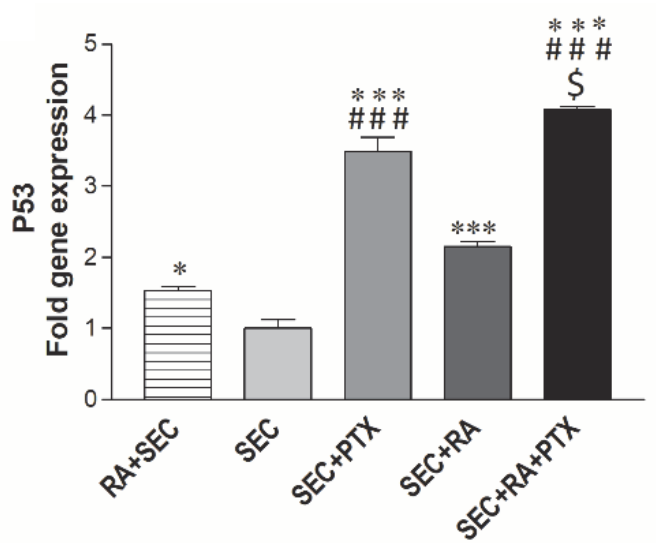

(a)



(b)

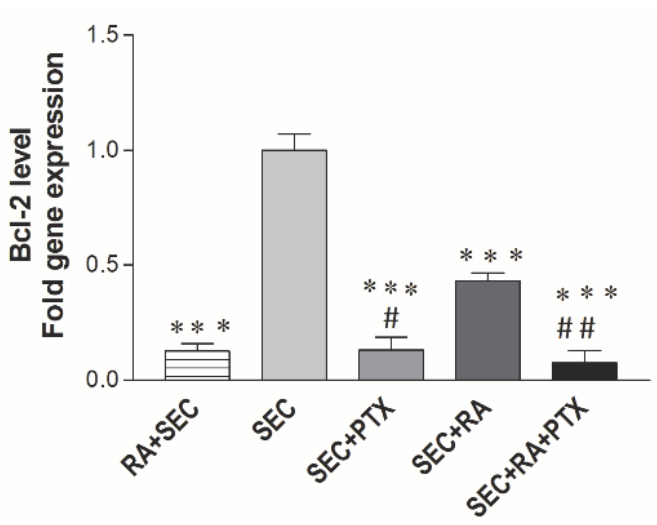

(c)

Fig. 3. mRNA expression of $\mathrm{P} 53, \mathrm{Bcl} 2$ and Bax in Ehrlich solid tumor bearing mice using qRT-PCR with specific primers. Effect of RA, PTX and their combination on (a) P53 level, (b) Bax level, (c) Bcl-2 level. Quantification analysis of mRNA was normalized, with GAPDH used as the housekeeping gene. Data are expressed as the mean \pm SD $(n=6)$. Data were analyzed using one-way ANOVA followed by Tukey post-hoc test. RA: Rosmarinic acid; SEC: Solid Ehrlich Carcinoma; PTX: Paclitaxel. * Significant difference from SEC. \$ Significant difference from SEC + PTX group. \# Significant difference from SEC + RA group (\# or $\$$ represent $p<0.05,{ }^{* *}$ or \#\# represent $p<0.01,{ }^{* * *}$ represent $p<0.001$ ).
Moreover, there was a significant decrease in gene expression of Bcl-2 in both prophylactic and treated groups compared to SEC-bearing mice - as shown in (Fig. 3c). The percentage of down-regulations of Bcl-2 mRNA expressions of $\mathrm{RA}+\mathrm{SEC}, \mathrm{SEC}+\mathrm{PTX}, \mathrm{SEC}+\mathrm{RA}, \mathrm{SEC}+\mathrm{RA}+\mathrm{PTX}$ were $87.35 \%$, $86.73 \%, 56.38 \%$, and $92.38 \%$ respectively, compared to that of SEC-bearing mice.

The RA and PTX combination treated group showed the most significant increase in P53 and Bax mRNA expressions, and the highest decrease in Bcl2 mRNA expression. Fold gene expression is represented in Fig. 3.

\section{Histopathological examination and immunohistochemical examination}

Histopathological examination revealed marked histopathological changes in tumor tissue - as shown in Fig. 4. Mice given RA prophylactic and mice treated with RA + PTX showed a normal structure without signs of degeneration or cellular infiltration. In contrast, the untreated mice SEC group showed active tumor cells with undifferentiated epithelial cells, significant infiltration, and large hyper chromatic pleomorphic nuclei.

In addition, Mice treated with RA alone and PTX alone exhibited considerable improvement compared with the SEC group. This is evidenced by a regression of tumor development with zones of apoptotic cells, and cellular infiltration.

Immunohistochemical investigation revealed a strong positive reaction for P53 and Caspase-3 in mice that received RA for prophylaxis or a combination of PTX and RA for treatment. Meanwhile P53 and Caspase-3 showed mild reactivity in PTX or RA single treatment when compared with SEC as revealed in Figs $5 a$ and $5 b$.

The tumor section in SEC-bearing mice showed a strong positive reaction for NF- $\kappa \mathrm{B}$, VEGF receptor, and Bcl-2. There was only mild immunoreactivity in mice treated with prophylactic RA and PTX or RA as single agent with the least reactivity to NF- $\mathrm{B}$, VEGF receptor, and Bcl-2 in RA + PTX treated mice as revealed in Figs $6 \mathrm{a}, 6 \mathrm{~b}$, and $6 \mathrm{c}$.

\section{Discussion}

RA is a naturally occurring polyphenol, which was previously studied for different medicinal purposes (Anwar et al., 2020). In the present study, the chemo-preventive and therapeutic potential of RA was elucidated as a single agent and in combination with PTX in mice breast cancer model.

A low dose of oral RA (50 mg) two weeks prior to tumor induction could significantly suppress breast cancer growth in various ways: (a) stimulating apoptosis and restricting anti-apoptotic proteins expression, (b) restricting angiogenesis, and (c) regulating inflammatory responses. Its chemo-preventive effect may be through the inhibition of NF/ $\mathrm{kB}-\mathrm{P} 53-$ Caspase-3 pathway. In agreement with our findings, it was found that $100 \mathrm{mg} / \mathrm{kg}$ RA inhibits skin cancer progression when used one week prior to cancer induction in mice by restoring levels of caspases, P53, and Bcl-2 (Moore et al., 2016).

$\mathrm{RA}$ is used for the treatment of different types of cancers as it acts through different pathways including blockage of tumor cell proliferation, apoptosis, metastasis, and inflammation (Nadeem et al., 2019). Zhanjun Ma reported that RA promotes apoptosis and suppresses the Bcl-2/Bax ratio, mainly through mitochondrial apoptotic pathways on osteosarcoma cells and enhances caspases cleavage (Ma et al., 2020). Also observed were decreased expression and protein levels of Bcl-2/Bax after RA treatment in breast cancer (Li et al., 2019a). Similarly, this 

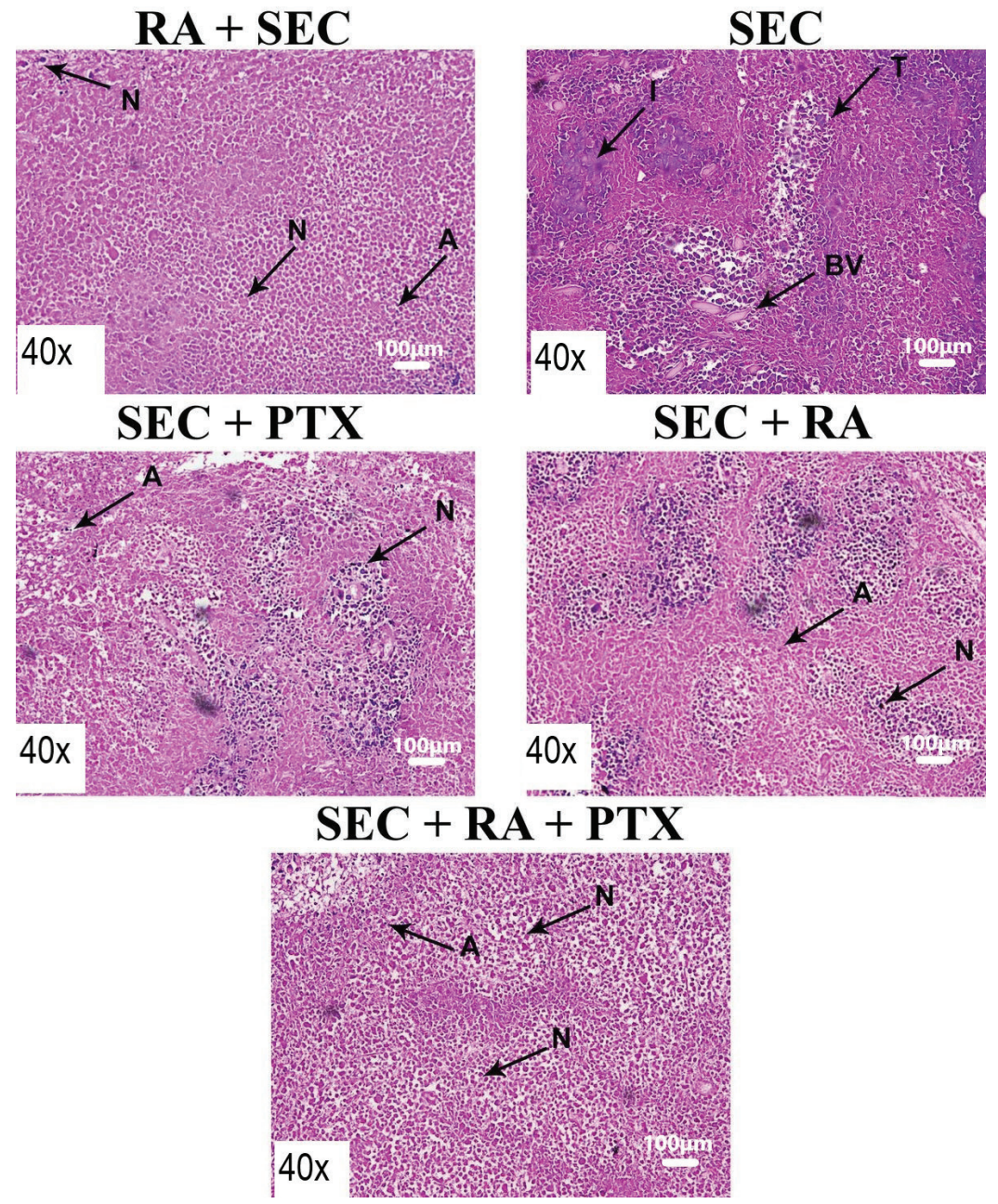

Fig. 4. Histopathological examination of solid tumor sections stained by hematoxylin and eosin ( $\times 40)$. RA: Rosmarinic acid; SEC: Solid Ehrlich Carcinoma; PTX: Paclitaxel; T: tumor cells; BV: blood vessel; I: infiltration; A: apoptotic parts; N: necrosis.

study suggests that RA inhibits tumor growth mainly through mitochondrial apoptotic pathways and caspase cleavage.

$\mathrm{NF}-\mathrm{\kappa B}$ is associated with many cancer pathways, including inflammation, angiogenesis, and apoptosis, suggesting its role in poor cancer prognosis and the development of drug resistance (Li et al., 2019b). RA was found to suppress the systemic release of many pro-inflammatory cytokines, mainly through the blockage of the NF- $\mathrm{kB}$ pathway (Fan et al., 2015). The levels of NF- $\mathrm{KB}$ correlates with the expression of VEGF in breast cancer. Therefore, the hindrance of NF- $\mathrm{kB}$ activation results in a decrease in VEGF mRNA expressions (Shibata et al., 2002). RA given either before or after tumor induction has an anti-angiogenesis effect by suppression of VEGF expression or protein levels (Değer and Çavuş, 2020).

In this experimental approach, PTX was found to have the ability to inhibit tumor growth in corroboration with previous studies. Similar findings were reported in acute lung injury in a mouse model; PTX inhibits NF- $\mathrm{BB}$ activation and inflammation (Wang et al., 2019). In addition, low dose PTX showed anti-angiogenetic effect by suppressing angiogenesis markers at protein and mRNA levels in breast cancer (Bocci et al., 2013). PTX enhances apoptosis in breast cancer via the activation of P53 expressions, resulting in caspase activation - mainly through the mitochondrial pathway and suppression of the Bcl2/Bax ratio (Ren et al., 2018).
A low dose of PTX in combination with other chemotherapy agents enhanced the apoptotic effect in breast cancer compared to PTX monotherapy (Clark et al., 2019). These observations strongly imply that RA can be used as an adjuvant therapy with PTX. Mice treated with a combination of RA and PTX showed the most significant reduction in tumor size. Co-treatment with RA and PTX had anti-inflammatory and antiangiogenic effects with a higher apoptotic rate than their monotherapies. This suggests that RA sensitizes breast cancer cells to PTX probably via the NF-kB-P53-Caspase-3 pathway.

\section{Conclusions}

RA shows good potential in inhibiting various hallmarks of the Ehrlich solid tumor. It is a promising substance that may be used as food additive or food supplement as prophylaxis for breast cancer. RA exerts antitumor effects in Ehrlich solid tumor through anti-inflammatory, apoptotic, and anti-angiogenic properties. This effect, when used in combination with PTX, was more efficient in comparison to PTX or RA monotherapies. Therefore, RA may provide new promise for agents that potentiate the antitumor activity of PTX. 

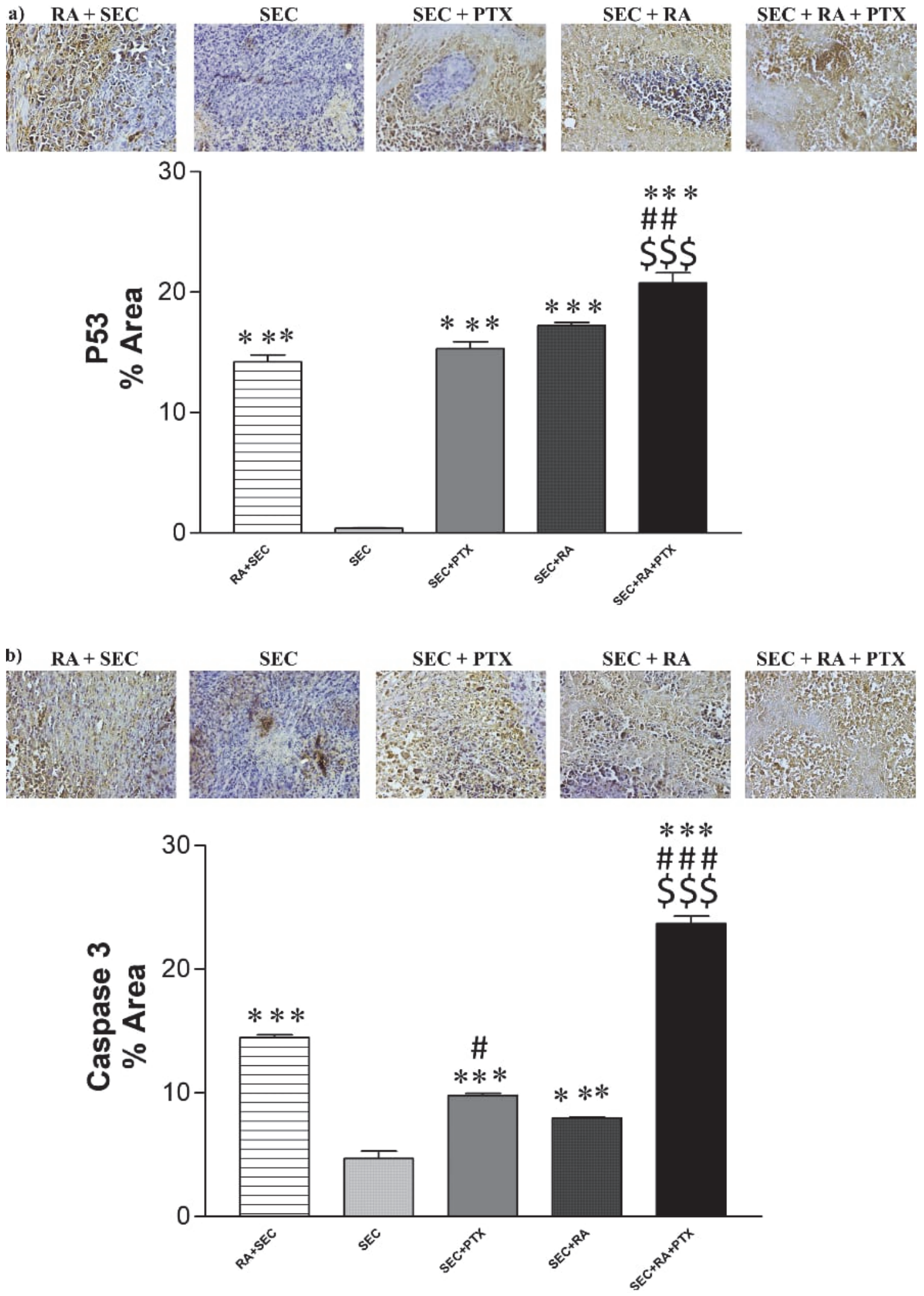

Fig. 5. Immunostaining and \% Area of P53 and Caspase-3 expression. (a) P53 and (b) Caspase-3 immunostaining images magnification at 40x and their \% area in the experimental groups. Data are expressed as the mean $\pm \operatorname{SD}(n=3)$. Data were analyzed using one-way ANOVA followed by Tukey post-hoc test. RA: Rosmarinic acid; SEC: Solid Ehrlich Carcinoma; PTX: Paclitaxel. * Significant difference from the SEC. \$ Significant difference from SEC + PTX group. \# Significant difference from SEC + RA group (\# represent $p<0.05$, \#\# represent $p<0.01$, ${ }^{* * *}$ or \#\#\# or \$\$ represent $p<0.001)$. 

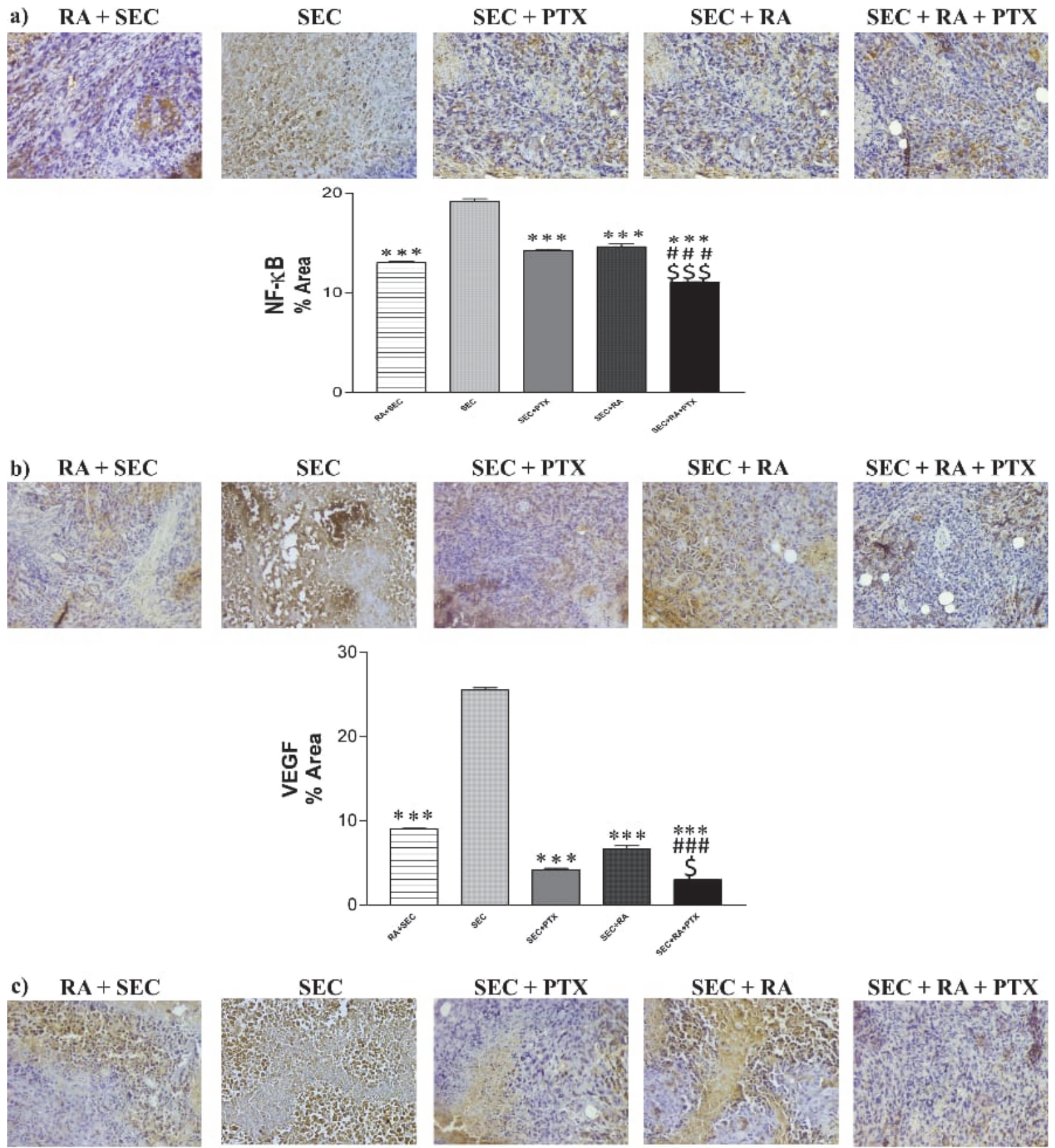

$$
\text { SEC + PTX }
$$

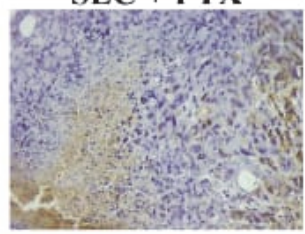

SEC + RA
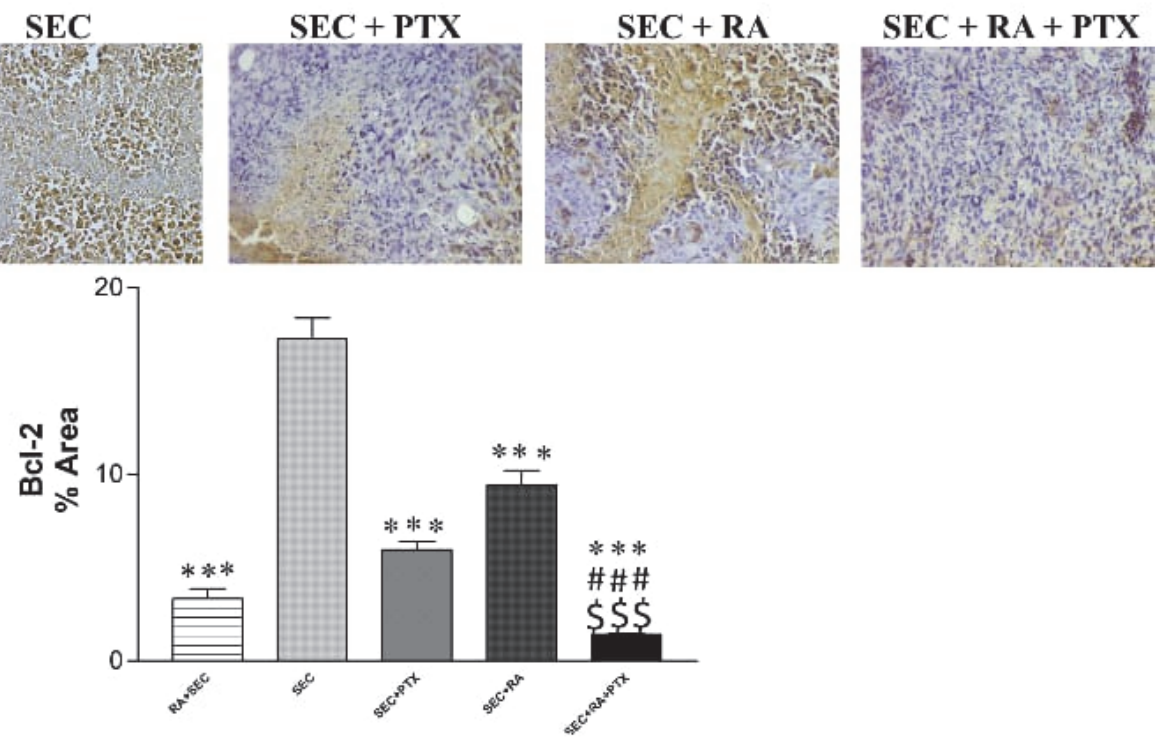

Fig. 6. Immunostaining and \% Area of NF- $\mathrm{B}$, VEGF receptor and Bcl-2 expression. (a) NF- $\mathrm{B}$, (b) VEGF receptor, and (c) Bcl-2 immunostaining images magnification at $40 \times$ and their $\%$ area in the experimental groups. Data are expressed as the mean $\pm S D(n=3)$. Data were analyzed using one-way ANOVA followed by Tukey post-hoc test. RA: Rosmarinic acid; SEC: Solid Ehrlich Carcinoma; PTX: Paclitaxel. * Significant difference from the SEC. \$ Significant difference from SEC+ PTX group. \# Significant difference from SEC + RA group $(\$$ represent $p<0.05$, *** or \#\#\# or $\$ \$$ represent $p<0.001)$. 


\section{Funding sources}

This research did not receive any specific grant from funding agencies in the public, commercial, or not-for-profit sectors.

\section{Ethical aspects and conflict of interests}

The authors report no conflict of interests.

\section{Author contributions}

Marwa A. Mahmoud: conceptualization, methodology and writing - original draft. Tark M. Okda: formal analysis, writing-review \& editing. Gamal A. Omran: supervision, validation, writing-review and editing. Mohammad M. Abd-Alhaseeb: conceptualization, data curation, formal analysis, software, validation, writing \& editing. All authors read and approved the final manuscript.

\section{References}

Abd-Alhaseeb MM, Zaitone SA, Abou-El-Ela SH, Moustafa YM (2013). Assessment of the Safety of Olmesartan in Combination with Sorafenib in Mice Bearing Ehrlich's Ascites Carcinoma. J Cancer Ther 4(8): 1355-1361. DOI: 10.4236/jct.2013.48160.

Abd-Alhaseeb MM, Zaitone SA, Abou-El-Ela SH, Moustafa YM (2014). Olmesartan Potentiates the Anti-Angiogenic Effect of Sorafenib in Mice Bearing Ehrlich's Ascites Carcinoma: Role of Angiotensin (1-7). PLoS One 9(1): e85891. DOI: 10.1371/journal. pone. 0085891 .

Al-Harras MF, Houssen ME, Shaker ME, Farag K, Farouk O, Monir R, et al. (2016). Polymorphisms of glutathione S-transferase $\pi 1$ and toll-like receptors 2 and 9: Association with breast cancer susceptibility. Oncol Lett 11(3): 2182-2188. DOI: 10.3892/ ol.2016.4159.

Anjum S, Anuradha A, Krishna A (2018). A possible direct action of oxytocin on spermatogenesis and steroidogenesis in pre-pubertal mouse. Andrologia 50(4): e12958. DOI: 10.1111/and.12958.

Anwar S, Shamsi A, Shahbaaz M, Queen A, Khan P, Hasan GM, et al. (2020). Rosmarinic Acid Exhibits Anticancer Effects via MARK4 Inhibition. Sci Rep 10(1): 1-13. DOI: 10.1038/s41598-02065648-z.

Bocci G, Di Paolo A, Danesi R (2013). The pharmacological bases of the antiangiogenic activity of paclitaxel. Angiogenesis 16(3): 481-492. DOI: 10.1007/s10456-013-9334-0.

Clark AS, McAndrew NP, Troxel A, Feldman M, Lal P, Rosen M, et al. (2019). Combination Paclitaxel and Palbociclib: Results of a Phase I Trial in Advanced Breast Cancer. Clin Cancer Res 25(7): 2072-2079. DOI: 10.1158/1078-0432.ccr-18-0790.

Day CM, Hickey SM, Song Y, Plush SE, Garg S (2020). Novel Tamoxifen Nanoformulations for Improving Breast Cancer Treatment: Old Wine in New Bottles. Molecules 25(5): 1182. DOI: 10.3390/molecules25051182.

Değer U, Çavuş Y (2020). Investigation of the role of rosmarinic acid treatment in regulating inflammation, cell damage, and angiogenesis in rat ovarian torsion and detorsion models. Acta Cir Bras 35(3): e202000304. DOI: 10.1590/s0102865020200030000004

Elufioye TO, Habtemariam S (2019). Hepatoprotective effects of rosmarinic acid: Insight into its mechanisms of action. Biomed Pharmacother 112: 108600. DOI: 10.1016/j.biopha.2019.108600.

Fan Y-T, Yin G-J, Xiao W-Q, Qiu L, Yu G, Hu Y-L, et al. (2015). Rosmarinic Acid Attenuates Sodium Taurocholate-Induced Acute Pancreatitis in Rats by Inhibiting Nuclear Factor- $\kappa B$ Activation. Am J Chin Med 43(6): 1117-1135. DOI: 10.1142/ s0192415x15500640.

Feitosa IB, Mori B, Garcia Teles CB, da Costa AG (2020). What are the immune responses during the growth of Ehrlich's tumor in ascitic and solid form? Life Sci 264: 118578. DOI: 10.1016/j. lfs.2020.118578.

Ghoneum M, Badr El-Din NK, Mahmoud AZ, Tolentino L, Pan D, Hassan TA (2019). Dietary baker's yeast sensitizes Ehrlich mammary adenocarcinoma to paclitaxel in mice bearing tumor. Oncol Rep 41(6): 3155-3166. DOI: 10.3892/or.2019.7107.

Han Y-H, Kee J-Y, Hong S-H (2018). Rosmarinic acid activates AMPK to inhibit metastasis of colorectal cancer. Front Pharmacol 9: 68. DOI: 10.3389/fphar.2018.00068.

Kang HJ, Lee SH, Price JE, Kim LS (2009). Curcumin Suppresses the Paclitaxel-Induced Nuclear Factor- $\kappa B$ in Breast Cancer Cells and Potentiates the Growth Inhibitory Effect of Paclitaxel in a Breast Cancer Nude Mice Model. Breast J 15(3): 223-229. DOI: 10.1111/j.1524-4741.2009.00709.x.

Katary MA, Abdelsayed R, Alhashim A (2019). Salvianolic Acid B Slows the Progression of Breast Cancer Cell Growth via Enhancement of Apoptosis and Reduction of Oxidative Stress, Inflammation, and Angiogenesis. Int J Mol Sci 20(22): 5653. DOI: 10.3390/ijms20225653.

Kirchler CG, Pezzei CK, Beć KB, Henn R, Ishigaki M, Ozaki Y, Huck CW (2017). Critical evaluation of NIR and ATR-IR spectroscopic quantifications of rosmarinic acid in rosmarini folium supported by quantum chemical calculations. Planta Med 83(12-13): 1076-1084. DOI: 10.1055/s-0043-107032.

Li H, Zhang Y, Chen H-H, Huang E, Zhuang H, Li D, Ni F (2019a). Rosmarinic acid inhibits stem-like breast cancer through hedgehog and Bcl-2/Bax signaling pathways. Pharmacogn Mag 15(65): 600-606. DOI: 10.4103/pm.pm_22_19.

Li W, Li Q, Wei L, Pan X, Huang D, Gan J, Tang S (2019b). Rosmarinic Acid Analogue-11 Induces Apoptosis of Human Gastric Cancer SGC-7901 Cells via the Epidermal Growth Factor Receptor (EGFR)/Akt/Nuclear Factor kappa B (NF-кB) Pathway. Med Sci Monit Basic Res 25: 63-75. DOI: 10.12659/MSMBR.913331.

Livak KJ, Schmittgen TD (2001). Analysis of relative gene expression data using real-time quantitative PCR and the $2^{-\triangle \Delta C \mathrm{~T}}$ method. Methods 25(4): 402-408. DOI: 10.1006/meth.2001.1262.

Ma Z, Yang J, Yang Y, Wang X, Chen G, Shi A, et al. (2020). Rosmarinic acid exerts an anticancer effect on osteosarcoma cells by inhibiting DJ-1 via regulation of the PTEN-PI3K-Akt signaling pathway. Phytomedicine 68: 153186. DOI: 10.1016/j. phymed.2020.153186.

Moore J, Yousef M, Tsiani E (2016). Anticancer effects of rosemary (Rosmarinus officinalis L.) extract and rosemary extract polyphenols. Nutrients 8(11): 731. DOI: $10.3390 /$ nu8110731.

Nadeem M, Imran M, Aslam Gondal T, Imran A, Shahbaz M, Muhammad Amir R, et al. (2019). Therapeutic Potential of Rosmarinic Acid: A Comprehensive Review. Appl Sci 9(15): 3139. DOI: 10.3390/app9153139.

Parveen A, Subedi L, Kim HW, Khan Z, Zahra Z, Farooqi MQ, Kim SJ (2019). Phytochemicals Targeting VEGF and VEGFRelated Multifactors as Anticancer Therapy. J Clin Med 8(3): 350. DOI: $10.3390 / \mathrm{jcm} 8030350$

Ren X, Zhao B, Chang H, Xiao M, Wu Y, Liu Y (2018). Paclitaxel suppresses proliferation and induces apoptosis through regulation of ROS and the AKT/MAPK signaling pathway in canine mammary gland tumor cells. Mol Med Rep 17(6): 8289-8299. DOI: 10.3892/ mmr.2018.8868.

Rocha J, Eduardo-Figueira M, Barateiro A, Fernandes A, Brites D, Bronze R, et al. (2015). Anti-inflammatory Effect of Rosmarinic Acid and an Extract of Rosmarinus officinalis in Rat Models of Local and Systemic Inflammation. Basic Clin Pharmacol Toxicol 116(5): 398-413. DOI: 10.1111/bcpt.12335.

Shibata A, Nagaya T, Imai T, Funahashi H, Nakao A, Seo H (2002). Inhibition of NF- $\mathrm{kB}$ Activity Decreases the VEGF mRNA Expression in MDA-MB-231 Breast Cancer Cells. Breast Cancer Res Treat 73(3): 237-243. DOI: 10.1023/A:1015872531675.

Trócsányi E, György Z, Zámboriné-Németh É (2020). New insights into rosmarinic acid biosynthesis based on molecular studies. Curr Plant Biol 23: 100162. DOI: 10.1016/j.cpb.2020.100162.

Wang Y-M, Ji R, Chen W-W, Huang S-W, Zheng Y-J, Yang Z-T, et al. (2019). Paclitaxel alleviated sepsis-induced acute lung injury by activating MUC1 and suppressing TLR-4/NF- $\mathrm{BB}$ pathway. Drug Des Devel Ther 13: 3391. DOI: 10.2147/2FDDDT.S222296.

Xu H, Wang L, Shi B, Hu L, Gan C, Wang Y, et al. (2020). Caffeine inhibits the anticancer activity of paclitaxel via down-regulation of a-tubulin acetylation. Biomed Pharmacother 129: 110441. DOI: 10.1016/j.biopha.2020.110441. 